\title{
Isolated angioedema of the bowel caused by aspirin
}

\author{
Karim Osman ${ }^{1,2} \cdot$ Ayse Tuba Kendi $^{3} \cdot$ Daniel Maselli $^{1}$
}

Received: 10 February 2021 / Accepted: 29 April 2021 / Published online: 17 May 2021

(c) Japanese Society of Gastroenterology 2021

\begin{abstract}
Angioedema is a self-limited, localized tissue swelling, resulting from fluid extravasation into interstitial spaces. It may occur in isolation or be accompanied by urticaria and/or anaphylaxis. The phenomenon has been linked to multiple medications, including non-steroidal anti-inflammatory drugs (NSAIDs) and angiotensin-converting enzyme inhibitors (ACEIs). NSAID-induced angioedema is observed in $<0.3 \%$ of patients taking NSAIDs. While isolated visceral angioedema has been reported from ACEIs, it has not been documented from NSAID use, particularly aspirin usage. Here, we report a case of isolated visceral angioedema attributed to aspirin use.
\end{abstract}

Keywords Angioedema $\cdot$ Small bowel $\cdot$ Non-steroidal anti-inflammatory drugs $\cdot$ Aspirin

\section{Introduction}

Angioedema can affect multiple tissues, either as a diffuse, multi-organ process or in the tissue of a specific organ. Isolated visceral angioedema (VA) — the swelling of the bowel wall without systemic edema-has been observed in association with several medications, particularly with angiotensinconverting enzyme inhibitors (ACEIs) [1]. While non-steroidal anti-inflammatory drugs (NSAIDs) have been linked to angioedema [2], to date there has been no report of NSAIDs associated with isolated VA. In this report, we document a case of an 80-year-old woman with isolated VA suspected to be related to her consumption of $325 \mathrm{mg}$ of aspirin twicedaily, which would be a unique clinical entity within the published literature.

Karim Osman

Ktarek.osman@gmail.com

1 Division of Gastroenterology and Hepatology, Mayo Clinic, 200 1st St. SW, Rochester, MN 55902, USA

2 Department of Internal Medicine, Beth Israel Lahey Clinic, Burlington, MA 01803, USA

3 Department of Radiology, Mayo Clinic, 200 1st St. SW, Rochester, MN 55902, USA

\section{Case report}

An 80-year-old woman presented to an outside institution with acute epigastric pain, nausea, and vomiting. Her medical history included end-stage renal disease secondary to ANCA-associated vasculitis, status post kidney transplant 13 years prior to current presentation (on tacrolimus and prednisone), the surgery for which had required a $2 \mathrm{~cm}$ small bowel resection and side-to-side anastomosis. She additionally carried a history of hysterectomy for localized uterine cancer, hypertension managed with lisinopril, and aspirin $325 \mathrm{mg}$ BID for lumbago. She had experienced multiple instances of similar abdominal pain, nausea, and vomiting over the preceding year which had been attributed to small bowel obstruction from post-surgical adhesions, which responded to conservative therapy.

At this presentation, a computed tomography (CT) scan showed diffuse moderate thickening of the small bowel, particularly at the distal ileum, as well as thickening in the transverse colon. A possible superimposed closed small bowel loop was described in the left upper quadrant, but no other transition points distally. She was managed with bowel rest and intravenous hydration and discharged a few days later when asymptomatic. 1 month later, while still asymptomatic, she underwent non-contrasted MRI for surveillance of a pancreatic cyst, and the entire bowel showed no dilation or wall thickening.

Two weeks after her MRI, she had recurrence of nausea, vomiting, and abdominal pain. A CT scan showed 
multiple dilated, fluid-filled loops of small bowel, with a possible transition point at the anastomotic site in the rightlower quadrant. Her symptoms resolved with conservative management.

Two months later, she presented to our hospital with a recurrence of the same symptoms. A CT scan showed wall thickening and increased mucosal enhancement of several stretches of dilated ileum/distal jejunum (Fig. 1), as well as new thickening in the rectosigmoid colon extending to the descending and the mid-transverse colon (Fig. 2). There was no transition point to suggest bowel obstruction. The splanchnic vasculature appeared patent. A pediatric colonoscope could not safely traverse the sigmoid colon due to sharp angulations, so a gastroscope was inserted anally to the level of the hepatic flexure at $50 \mathrm{~cm}$, revealing unremarkable mucosa (Fig. 3). A capsule endoscopy was not pursued due to the elevated risk of capsule retention from her surgical adhesion burden, and balloon-assisted enteroscopy to evaluate and sample the small bowel posed considerable risk for colon perforation given the technical challenges of her colonoscopy so was similarly avoided. She was managed with bowel rest and intravenous hydration and improved within
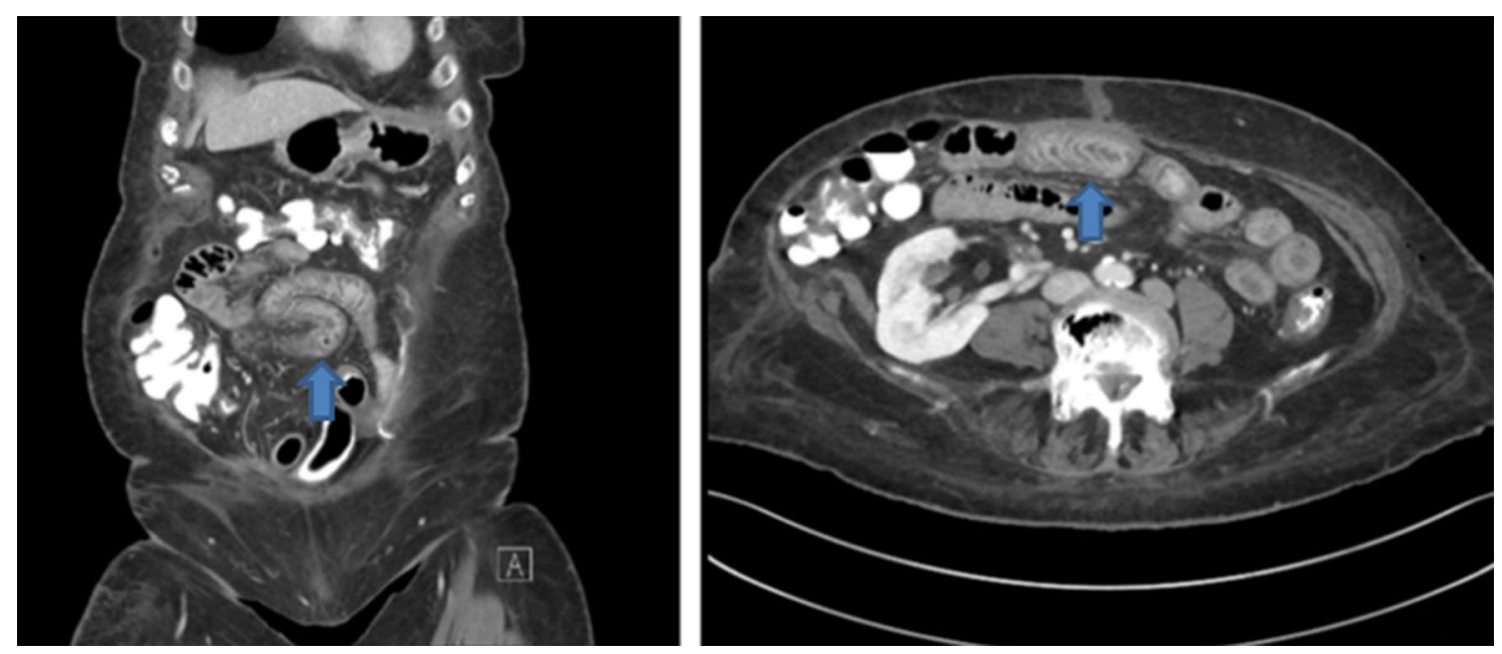

Fig. 1 Computed tomography (CT) scan of the abdomen with intravenous contrast showed wall thickening, edema and increased mucosal enhancement of multiple loops of the ileum and distal jejunum (arrows)
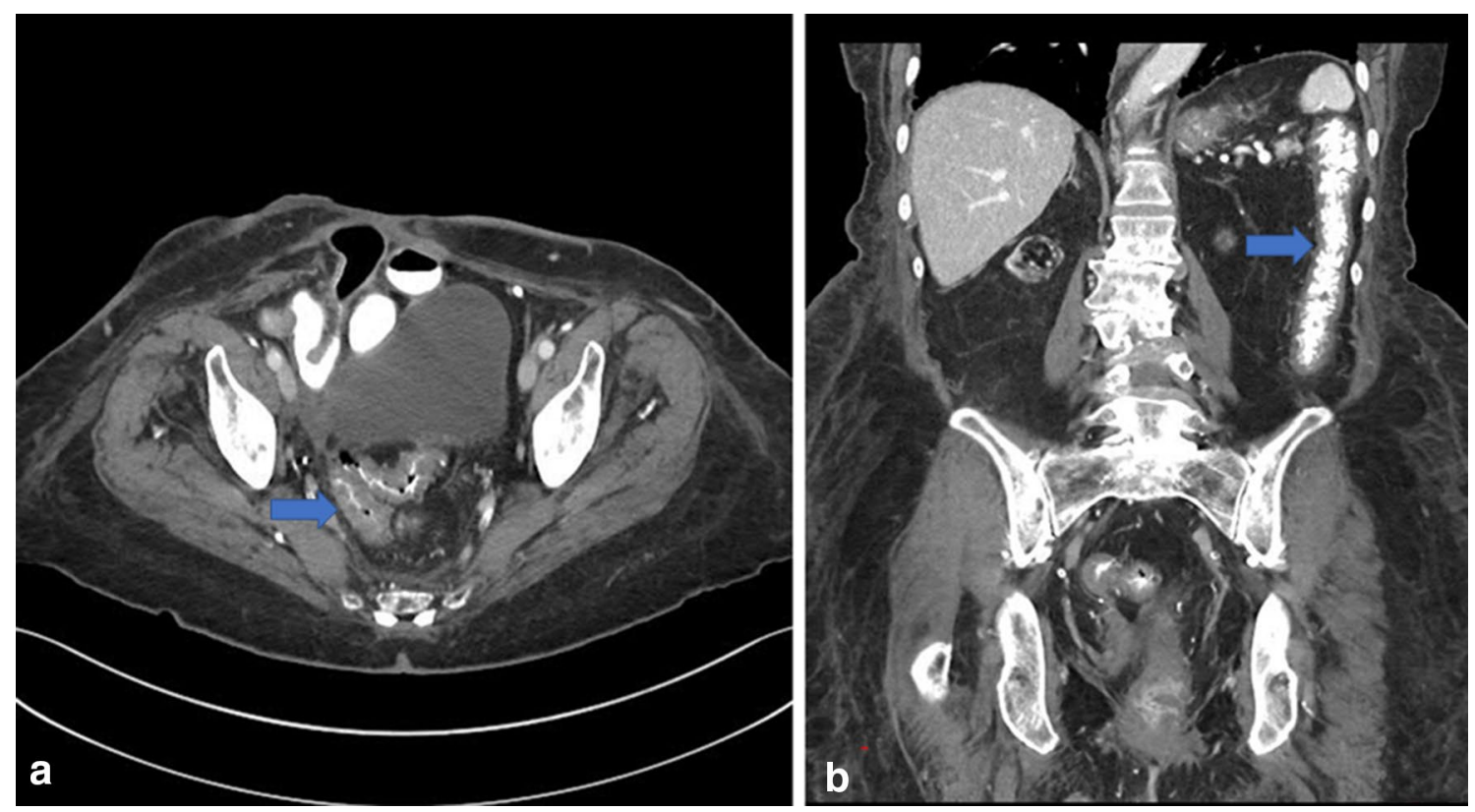

Fig. 2 Computed tomography (CT) scan of the abdomen with intravenous contrast showing thickening at rectosigmoid colon walls (a) and circumferential wall thickening at descending colon (b) 


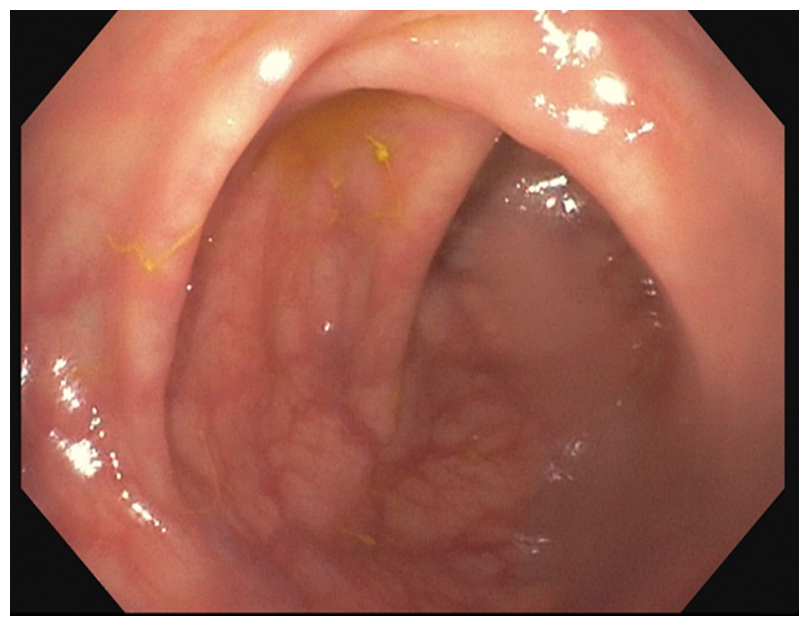

Fig. 3 Colonoscopy showing normal appearing mucosa with no mucosal edema or erythema

days. Given the absence of definitive bowel obstruction, as well as thickening of the bowel wall, it was thought she may have VA, thus her lisinopril was switched to amlodipine on discharge. Her aspirin, which was held during admission, was resumed.

One month later, she presented with the same symptoms, with a CT scan showing multiple areas of thickened walls of the small bowel (Fig. 4), as well as the colon, worsened from the prior examination, also with no transition point (Fig. 5). Evaluation showed unremarkable values for complete blood count with a normal absolute eosinophil count of $0.29 \times 10^{9} / \mathrm{L}$ (Normal range $0.03-0.48 \times 10^{9} / \mathrm{L}$ ), normal albumin level of $3.5 \mathrm{~g} / \mathrm{dL}$ (Normal range $3.5-5.0 \mathrm{~g} / \mathrm{dL}$ ), and normal liver function tests. C-Reactive Protein (CRP) was elevated at $28.7 \mathrm{mg} / \mathrm{L}$ (Normal range $<8.0 \mathrm{mg} / \mathrm{L}$ ). Serum complement $\mathrm{C} 4$ level and $\mathrm{C} 1$ esterase inhibitor functional assay were normal. Stool microscopy was negative for ova and parasites. Stool pathogen PCR panel was negative, as well, and serum cytomegalovirus DNA was unremarkable. The Kappa/Lambda free light chain ratio was within normal limits.

As there had been no ACEI exposure for the prior month, it was suspected that the trigger was her $325 \mathrm{mg}$ of aspirin, which was discontinued. The patient improved within $24 \mathrm{~h}$ and was discharged. Five weeks later, while asymptomatic, she underwent a CT scan with oral contrast, which showed resolution of the bowel wall thickening (Fig. 6).

The patient was scheduled to follow up with us, however, she was unable to since it was the peak of the COVID-19 pandemic. She did, however, have a telehealth visit with her transplant nephrologist, 6 months after discharge, where she stated that her bowel symptoms have greatly improved and that there was no recurrence of symptoms. Two months later after the telehealth visit, she unfortunately developed pneumonia secondary to COVID-19 infection and passed away.

Notably, in no episode described did the patient experience constitutional symptoms, dermatologic changes, or dyspnea/wheezing/hemoptysis. Nor did she have evidence of worsening renal function, hematuria, or end-organ dysfunction. She had no exposure to new medications, foreign travel, or dietary changes.
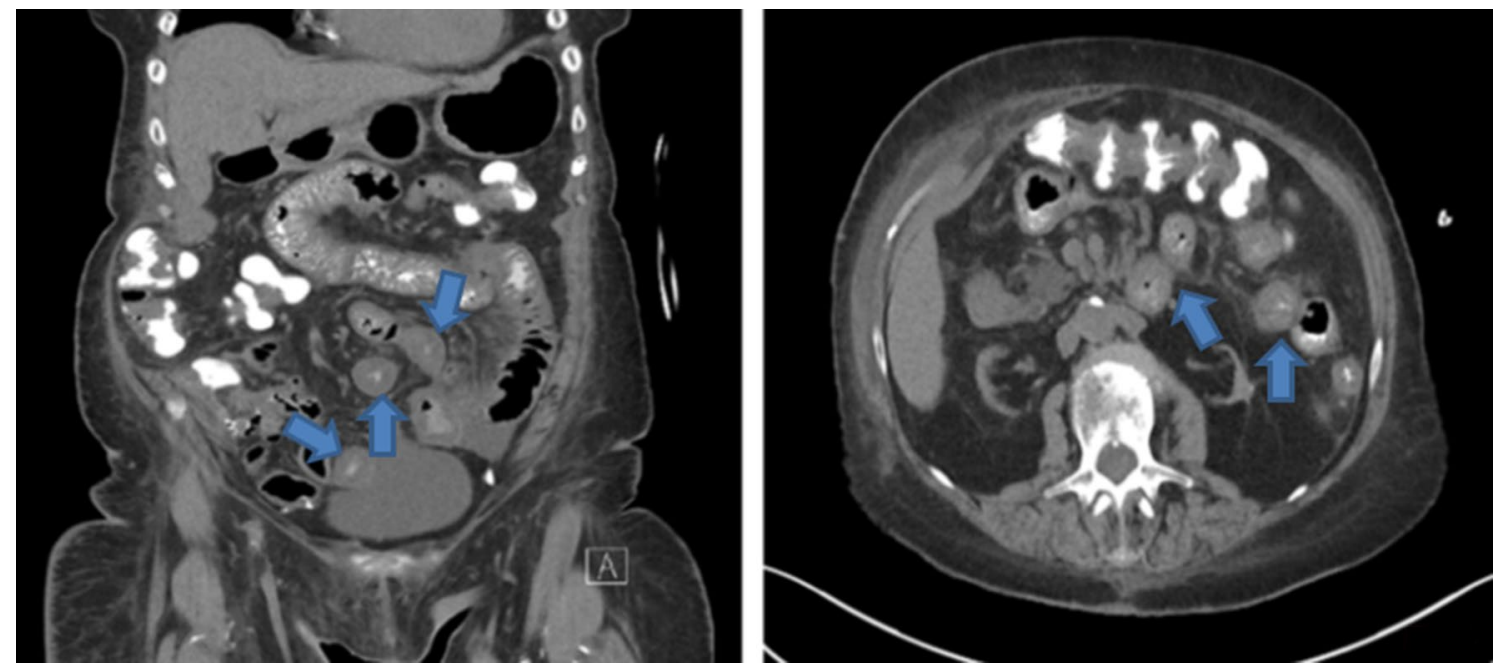

Fig. 4 Computed tomography (CT) scan of the abdomen with intravenous contrast showing similar findings with interval worsening of bowel wall thickening (arrows) compared to the previous CT scan. Diffuse mesenteric and visceral edema can be appreciated 


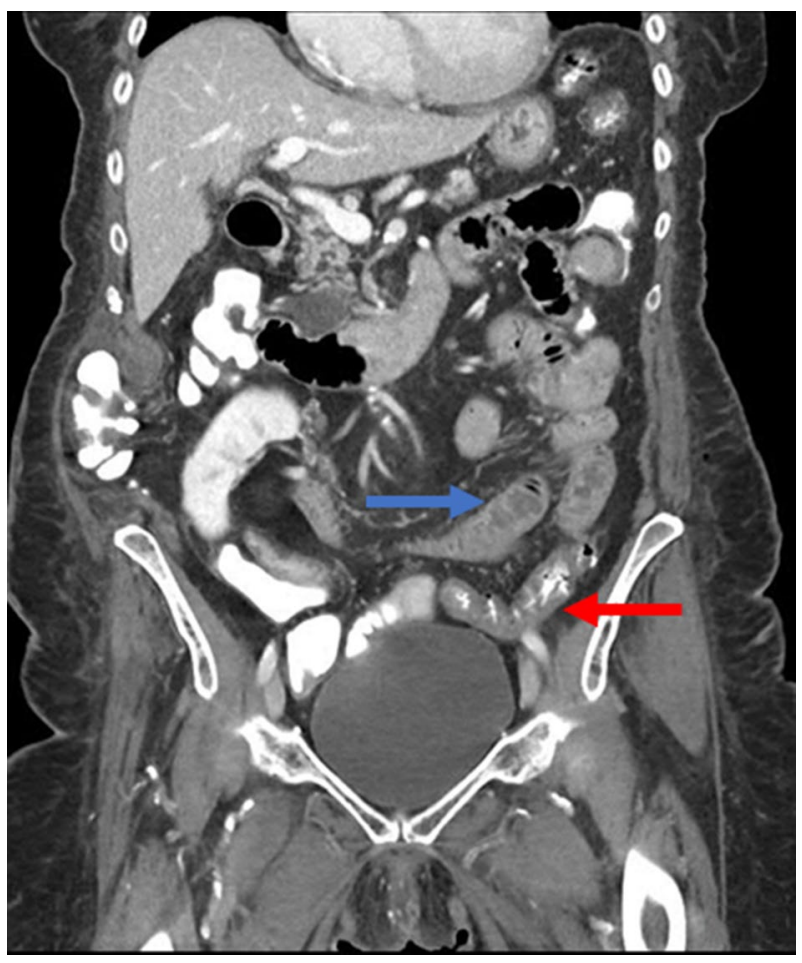

Fig. 5 Computed tomography (CT) scan of the abdomen with intravenous contrast shows worsening wall thickening at both the colon (red arrow) and the small bowel walls (blue arrow)

\section{Discussion}

NSAIDs are the most commonly prescribed analgesic and anti-inflammatory medication, accounting for $5-10 \%$ of all drugs prescribed annually [3]. NSAID, including aspirin, usage in the United States (US) continues to increase [4]. As such, clinicians must be aware of the spectrum of their adverse effects. These include a host of gastrointestinal phenomena, primarily manifesting as erosions, ulcerations, and strictures [5]. To enhance clinician's knowledge of additional effects, we present a case of recurrent isolated VA that was attributed to aspirin use.

Angioedema of the bowel typically presents with recurrent episodes of abdominal pain, nausea, and vomiting, similar to the presentations described in this case. The absence of cutaneous, oral, or respiratory symptoms should not preclude the diagnosis of angioedema, as gastrointestinal manifestations can predate the other symptoms by several years or can be the sole complaint $[1,6]$. Multidetector CT scan is the imaging of choice. The hallmark findings include localized circumferential bowel wall thickening-typically without frank obstruction - as well as mesenteric edema, and ascites. The length of affected bowel typically ranges from 20 to 60 $\mathrm{cm}$ and has a wall thickness range of 4-9 $\mathrm{mm}$ [6].

Beyond isolated VA, the differential diagnosis of multifocal visceral wall thickening without frank obstruction includes vasculitis, ischemia, Crohn's disease, infection, lymphoproliferative diseases, and radiation enteritis [6]. Many etiologies ultimately rely on histologic evidence, however, access to the affected portion of small bowel in our patient had significant technical limitations due to the patient's anatomy, and unfortunately no biopsies were taken of the affected colon at the time of endoscopic observation. Our clinical reasoning, therefore, is composed from patterns from the patient's history, physical exam, macroscopic endoscopic appearance, laboratory parameters, and clinical trajectory in the absence of additional treatments. Vasculitides-such as eosinophilic granulomatosis with polyangiitis, microscopic polyangiitis-induced enteritis, IgA vasculitis, and lupus enteritis - can often be ruled out by the lack of associated clinic and immunologic criteria [7]. Our patient notably lacked multisystem involvement that characterizes these disorders. Eosinophilic granulomatosis with polyangiitis is frequently triggered by allergens or infections. Nearly $100 \%$ of patients have a diagnosis of asthma and the mean age of diagnosis is $50 \pm 16$ years. Patients typically have peripheral eosinophilia and a normal CRP $[8,9]$. Our patient did not have a history of asthma, presented with
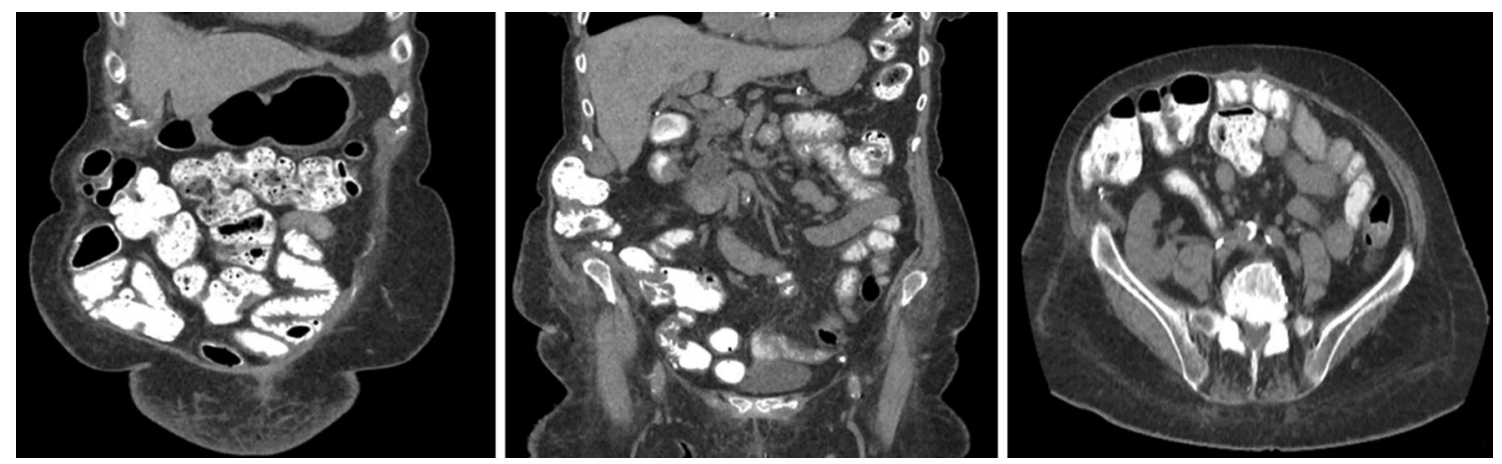

Fig. 6 Five weeks after discontinuing her twice-daily aspirin, the patient underwent computed tomography (CT) with oral (no IV) contrast, which showed interval resolution of the small bowel and colon wall thickening 
those symptoms at the age of 80 and her laboratory results argued against this diagnosis. Microscopic polyangiitisinduced enteritis is a necrotizing small vessel vasculitis and would be expected to show ulcerations of the small bowel from compromised vascular supply (no such findings were observed in the affected colon endoscopically). While gastrointestinal involvement is common in IgA vasculitis, it is relatively less common in the elderly population. Furthermore, it is typically accompanied with the characteristic palpable symmetrical purpura across pressure-dependent areas-an observation which is absent in our patient as well. Erythema, petechia, and ulcers are commonly seen in colonoscopy [10]. Systemic lupus erythematosus is an autoimmune multisystem disorder that typically affects young to middle aged women. It would be uncommon to present in the 7th-8th decades of life without any new cytopenia, dermatologic symptoms or renal involvement. Furthermore, if vasculitis were the etiology, we would have expected to observe ischemic enterocolitis or ulcerations in the colonoscopy from the compromised blood supply. We did not manage the patient with additional immunosuppression, and thus, it would be unexpected for the patient to have such rapid symptomatic resolution if the proposed etiology is vasculitis [11].

Given the lack of frank ulcerations of bowel mucosa, micro-inflammatory disorders were also in our differential. Eosinophilic gastroenteritis, for instance, can present with similar symptoms; however, it most commonly affects the stomach, followed by the small bowel, and rarely affects the colon. Patients typically have history of seasonal or food allergies, or asthma; and laboratory findings while non-specific, include eosinophilia, hypoalbuminemia, and elevated liver function tests. The symptoms are typically improved with discontinuation of inciting allergens and/or administering corticosteroids [12]. Our patient lacked any of these features. Microscopic enteritis would more commonly affect the small bowel and would be unlikely to show pronounced changes on cross sectional imaging or endoscopy with bowel wall thickening and edema, as the condition is characterized by microscopic and submicroscopic changes of the tissue.

The patency of mesenteric vessels and lack of a history of mesenteric insufficiency helps rule out visceral ischemia. The absence of stricturing or penetrating bowel disease, as well as frequent episodic presentation and resolution without immunosuppression point away from Crohn's disease. The absence of diarrhea, fever, lymphadenopathy, and identified pathogen in the stool point away from infectious etiologies. Finally, we did consider lymphoma, multiple myeloma, Waldenström's macroglobulinemia, and chronic lymphocytic leukemia part of our differential. However, our patient had a normal complete blood count and differential, no B symptoms (fever, night sweats, and weight loss), lymphadenopathy, or splenomegaly. The Kappa/Lambda free light chain ratio was within normal limits, as well.

Within isolated VA, etiologies include $\mathrm{C} 1$ inhibitor deficiency, which can be hereditary or acquired and is assessed with a serologic functional assay [13]. If not present, medication-associated VA should be suspected. ACEIs are by far the most commonly observed medications associated with angioedema, though other medication associations, such as with sirolimus, have been reported in the literature [14].

While ACEIs are thought to the most common etiology of isolated VA, the prevalence of ACEI-induced VA is imprecisely appreciated due to the paucity of overall cases of isolated VA. It is thought to be independent of the duration of ACEIs intake, as ACEI-induced VA has been reported from 2 days to 10 years from the start of on-going ACEI therapy $[1,6]$. While there is no gold standard in diagnosing the ACEI-induced VA, a consistent clinical picture that resolves within 24-48 h after discontinuation of ACEI supports the diagnosis. This timeline stands to reason given the half-life of lisinopril-the most commonly prescribed ACEI in the

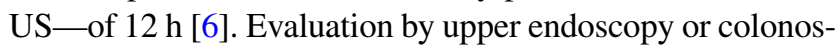
copy is usually unremarkable, though there have been some reports of using balloon-assisted enteroscopy to evaluate the distal small bowel, which is the usual site of involvement of drug-induced VA [15]. Beltrami et al. [16] showed a $46 \%$ recurrence rate of general angioedema after ACEI discontinuation, however, out of the 111 patients in that cohort, there was no recurrence of VA after ACEI discontinuation.

Other authors have suggested the possibility of other drugs instigating VA and thereby confounding the interpretation of recurrence of VA [17]. It is plausible, given their widespread use, that concurrent aspirin use may have contributed to these scenarios and led to an underappreciation of alternate pharmacologic causes of angioedema generally and VA specifically $[18,19]$. To date, there has been no report in the literature of aspirin causing VA, which we aim to amend through presentation of this case.

Ostensibly aspirin-induced VA follows similar mechanistic underpinnings of NSAID-induced angioedema (NIA). NIA presents in $0.1-0.3 \%$ of the population taking NSAIDs and has been reported to principally affect the face and be accompanied by urticaria [2]. It is thought to arise from inhibition of cyclooxygenase-1 (COX-1), which leads to preferential direction of arachidonic acid to the lipoxygenase pathway, prompting an increase in production of vasoactive leukotrienes responsible for increased capillary permeability, fluid extravasation, and edema [13]. One of the leading factors involved in the pathogenesis of NSAIDinduced enteropathy (NIE) is the inhibition of COX-1 and the attendant prostaglandin deficiency [20]. The shared pathologic mechanism of NIA and NIE may help explain why NSAIDs and aspirin have a predilection for small bowel 
Table 1 Clues for diagnosing NSAID-induced visceral angioedema

\author{
Acute episodic presentation of abdominal pain, nausea and vomiting \\ CT abdomen with contrast showing signs of bowel wall thickening, potentially with upstream dilation \\ No transition point seen on CT \\ Interval clinical improvement paralleling radiologic improvement of bowel thickening \\ Normal C4 level and C1 esterase inhibitor functional assay \\ Absence of alternative diagnoses \\ Symptom resolution after discontinuation of NSAIDs
}

manifestations, though it is unclear why patients may have NIE instead of NIA.

To fully make a diagnosis of aspirin-induced VA, other bowel-related pathology from NSAIDs/aspirin exposure need to be excluded. It is unlikely for our patient to have had NIE, since in addition to the pathogenesis mentioned earlier, there is a major COX-independent factor contributing to the development of NIE, which is the topical effect of NSAIDs on the mitochondria of the intestinal epithelial cells [21, 22]. Unlike other NSAIDs, aspirin cannot exert this topical effect since it is immediately absorbed in the stomach and duodenum and thus, it typically does not cause ileitis [21-23]. Collagenous colitis form NSAIDs was considered but is not associated with radiologic changes seen here and is typically associated with diarrhea rather than episodic obstructive symptoms [24, 25]. NSAID associated ileitis/colitis is commonly associated with mucosal surface changes, leading to erosions and ulcerations, which were not observed on the colonoscopy. While these entities can be associated with obstructive symptoms, this is characteristically from stricturing bowel disease, which would not have abrupt symptomology and radiologic onset and resolution [26].

This case highlights several critical learning points for clinical practice. First, this patient's diagnosis was delayed due to anchoring on small bowel obstruction, given the patient's surgical history, even when no obvious transition point was identified and bowel thickening occurred distal to instances where a putative obstruction was suspected. Second, the diagnosis of VA was considered and attributed to ACEIs use. This was a reasonable instinct, as isolated VA is most commonly associated with ACEIs use; however, when the VA recurred 1 month after discontinuation of lisinopril, the literature suggests that alternate causes should be explored. Finally, after considering the above differential for bowel thickening - and noting the oscillating pattern of obstructive symptoms associated with bowel edema and resolution of symptoms associated with radiologic normalization of the bowel, which illustrates the cadence of isolated VA-it was determined that the patient's NSAIDs were the most likely cause of her recurrent episodes of VA; nevertheless, histologic appraisal of affected tissue when feasible would enhance the diagnostic evaluation considerably in such cases. Table 1 highlights the teaching pearls for making a diagnosis of NSAID-induced VA.

Acknowledgements None.

Author contributions $\mathrm{KO}$ reviewed the literature and drafted the manuscript. ATK and DM reviewed the literature and edited the manuscript.

Funding None.

\section{Declarations}

Conflict of interest The authors declare that there is no conflict of interest.

\section{References:}

1. Scheirey CD, Scholz FJ, Shortsleeve MJ, et al. Angiotensin-converting enzyme inhibitor-induced small-bowel angioedema: clinical and imaging findings in 20 patients. AJR Am J Roentgenol. 2011;197:393-8.

2. Nzeako UC. Diagnosis and management of angioedema with abdominal involvement: a gastroenterology perspective. World J Gastroenterol. 2010;16:4913-21.

3. Wongrakpanich S, Wongrakpanich A, Melhado K, et al. A comprehensive review of non-steroidal anti-inflammatory drug use in the elderly. Aging Dis. 2018;9:143-50.

4. Davis JS, Lee HY, Kim J, et al. Use of non-steroidal anti-inflammatory drugs in US adults: changes over time and by demographic. Open Heart. 2017;4:e000550.

5. Lim YJ, Yang $\mathrm{C}-\mathrm{H}$. Non-steroidal anti-inflammatory drug-induced enteropathy. Clin Endosc. 2012;45:138-44.

6. Vallurupalli K, Coakley KJ. MDCT features of angiotensin-converting enzyme inhibitor-induced visceral angioedema. AJR Am J Roentgenol. 2011;196:W405-11.

7. Gross WL, Trabandt A, Reinhold-Keller E. Diagnosis and evaluation of vasculitis. Rheumatology. 2000;39:245-52.

8. Comarmond C, Pagnoux C, Khellaf M, et al. Eosinophilic granulomatosis with polyangiitis (Churg-Strauss): clinical characteristics and long-term followup of the 383 patients enrolled in the French Vasculitis Study Group cohort. Arthritis Rheum. 2013;65:270-81.

9. Sablé-Fourtassou R, Cohen P, Mahr A, et al. Antineutrophil cytoplasmic antibodies and the Churg-Strauss syndrome. Ann Intern Med. 2005;143:632-8.

10. Cojocariu C, Singeap AM, Chiriac S, et al. Gastrointestinal manifestations of IgA vasculitis-Henoch-Schönlein Purpura. Digestive system-recent advances. IntechOpen; 2019. 
11. Hokama A, Kishimoto K, Ihama Y, et al. Endoscopic and radiographic features of gastrointestinal involvement in vasculitis. World journal of gastrointestinal endoscopy. 2012;4:50-6.

12. Mori A, Enweluzo C, Grier D, et al. Eosinophilic gastroenteritis: review of a rare and treatable disease of the gastrointestinal tract. Case Rep Gastroenterol. 2013;7:293-8.

13. Banerji A. Hereditary angioedema: classification, pathogenesis, and diagnosis. Allergy Asthma Proc. 2011;32:403-7.

14. Yang H, Wang W, Hu X, et al. Sirolimus-induced severe small bowel angioedema: a case report. Medicine (Baltimore). 2018;97:e12029.

15. Spahn TW, Grosse-Thie W, Mueller MK. Endoscopic visualization of angiotensin-converting enzyme inhibitor-induced small bowel angioedema as a cause of relapsing abdominal pain using double-balloon enteroscopy. Dig Dis Sci. 2008;53:1257-60.

16. Beltrami L, Zanichelli A, Zingale L, et al. Long-term follow-up of 111 patients with angiotensin-converting enzyme inhibitor-related angioedema. J Hypertens. 2011;29:2273-7.

17. Guyer AC, Banerji A. ACE inhibitor-induced angioedema. In: UpToDate; 2015.

18. Banerji A, Blumenthal KG, Lai KH, et al. Epidemiology of ACE inhibitor angioedema utilizing a large electronic health record. J Allergy Clin Immunol Pract. 2017;5:744-9.

19. Wong JT, Hsu Y, Chen HJ, et al. Severe angioedema: Interaction among ACEI, ASA/NSAID, narcotics, and other contributing factors. J Allergy Clin Immunol. 2002;109:S128.
20. Takeuchi K, Satoh H. NSAID-induced small intestinal damageroles of various pathogenic factors. Digestion. 2015;91:218-32.

21. Somasundaram S, Sigthorsson G, Simpson R, et al. Uncoupling of intestinal mitochondrial oxidative phosphorylation and inhibition of cyclooxygenase are required for the development of NSAIDenteropathy in the rat. Aliment Pharmacol Ther. 2000;14:639-50.

22. Watanabe T, Fujiwara Y, Chan FKL. Current knowledge on nonsteroidal anti-inflammatory drug-induced small-bowel damage: a comprehensive review. J Gastroenterol. 2020;55:481-95.

23. Bjarnason I, Hayllar J, MacPherson AJ, et al. Side effects of nonsteroidal anti-inflammatory drugs on the small and large intestine in humans. Gastroenterology. 1993;104:1832-47.

24. Milman N, Kraag G. NSAID-induced collagenous colitis. J Rheumatol. 2010;37:2432.

25. Rasmussen MA, Munck LK. Systematic review: are lymphocytic colitis and collagenous colitis two subtypes of the same diseasemicroscopic colitis? Aliment Pharmacol Ther. 2012;36:79-90.

26. Geramizadeh B, Taghavi A, Banan B. Clinical, endoscopic and pathologic spectrum of non-steroidal anti-inflammatory druginduced colitis. Indian J Gastroenterol. 2009;28:150-3.

Publisher's Note Springer Nature remains neutral with regard to jurisdictional claims in published maps and institutional affiliations. 